\title{
Ensemble Learning for Human Activity Recognition
}

\author{
Ryoichi Sekiguchi \\ Tokyo Denki University \\ 5 Senju-Asahi-cho, Adachi-ku \\ Tokyo 120-8551, Japan \\ 20jkm17@ms.dendai.ac.jp
}

\author{
Kenji Abe \\ Tokyo Denki University \\ 5 Senju-Asahi-cho, Adachi-ku \\ Tokyo 120-8551, Japan \\ 20jkm02@ms.dendai.ac.jp
}

\author{
Takumi Yokoyama \\ Tokyo Denki University \\ 5 Senju-Asahi-cho, Adachi-ku \\ Tokyo 120-8551, Japan \\ 20jkm33@ms.dendai.ac.jp
}

\author{
Masayasu Kumano \\ Tokyo Denki University \\ 5 Senju-Asahi-cho, Adachi-ku \\ Tokyo 120-8551, Japan \\ 17aj057@ms.dendai.ac.jp
}

\author{
Masaki Kawakatsu \\ Tokyo Denki University \\ 5 Senju-Asahi-cho, Adachi-ku \\ Tokyo 120-8551, Japan \\ kawakatu@mail.dendai.ac.jp
}

\begin{abstract}
This paper describes a activity recognition method for SussexHuawei Locomotion (SHL) Challenge 2020 by team TDU_BSA. The use of ensemble learning, which combines the outputs of multiple classifiers to produce a single estimation result, improved the accuracy of activity recognition. The ensemble model consists of CNN models and a gradient-boosting model. The objective of SHL Challenge 2020 is that the users of SHL test-set are two different from SHL training-set, and the phone location of SHL test-set is not known to the SHL's participants. Therefore, estimating phone location and the user improved accuracy. SHL test-set's phone location was estimated to be Hips. The user can be estimated from SHL validation-set. The ensemble model was made with all SHL training-set (Only Hips) and 70\% of SHL validation-set (Only Hips). In the submission phase, the best F-measure obtained for last $30 \%$ SHL validation-set was $84.8 \%$.
\end{abstract}

\section{CCS CONCEPTS}

- Computing methodologies $\rightarrow$ Activity recognition and understanding.

\section{KEYWORDS}

Ensemble learning; CNN; Time-Frequency analysis; User estimation; Phone location estimation

\section{ACM Reference Format:}

Ryoichi Sekiguchi, Kenji Abe, Takumi Yokoyama, Masayasu Kumano, and Masaki Kawakatsu. 2020. Ensemble Learning for Human Activity Recognition. In Adjunct Proceedings of the 2020 ACM International foint Conference on Pervasive and Ubiquitous Computing and Proceedings of the 2020 ACM International Symposium on Wearable Computers (UbiComp/ISWC '20 Adjunct), September 12-16, 2020, Virtual Event, Mexico. ACM, New York, NY, USA, 5 pages. https://doi.org/10.1145/3410530.3414346

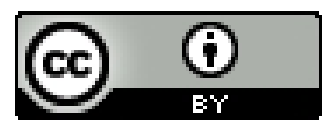

This work is licensed under a Creative Commons Attribution International 4.0 License.

UbiComp/ISWC '20 Adjunct, September 12-16, 2020, Virtual Event, Mexico

(c) 2020 Association for Computing Machinery.

ACM ISBN 978-1-4503-8076-8/20/09 .. \$15.00

https://doi.org/10.1145/3410530.3414346

\section{INTRODUCTION}

Many studies have been conducted to estimate the activity from sensors mounted on a smartphone. The authors participated in Sussex-Huawei Locomotion (SHL) Challenge 2020 as a team TDU_B SA. Our goal is to develop a activity recognition algorithm using the SHL dataset $2020[1,2]$.

In machine learning, ensemble learning that combines multiple classifiers and integrates the results to improve estimation accuracy is more effective than individual classifiers. A logistic regression model was made with the output probabilities of five classifiers as features. For train and subway, we used the results of models that were not used as input for the logistic regression model. The final estimation results were obtained.

\section{SHL CHALLENGE DATA}

SHL Challenge set was measured by four smartphones that were put on different positions (Bag, Hand, Hips, and Torso).

The SHL Challenge set consists of SHL training-set, SHL validationset, and SHL test-set. SHL training-set had collected by User-1 for 59 days. Moreover, SHL validation-set had collected by User- 2 and User-3 for six days. Both the datasets have four different phone positions. SHL test-set had collected by User- 2 and User-3 for 40 days, but it is at one unknown location.

These datasets were sampled at $100 \mathrm{~Hz}$ and has 9 sensors. They are acceleration sensor (Acc), gravity sensor (Gra), gyroscope sensor (Gyr), linear acceleration sensor (LAcc), geomagnetic sensor (Mag), orientation sensor (Ori) and atmospheric pressure sensor (Pre). Acc, Gra, Gyr, LAcc, and Mag has axis x, y, and z. The sensor Ori has axis $\mathrm{x}, \mathrm{y}, \mathrm{z}$, and $\mathrm{w}$.

These datasets were segmented by five seconds time-window, labeled every frame. Labels are "Still," "Walking," "Run," "Bike," "Car," "Bus," "Train," and "Subway." The objective of this challenge is to classify these data into these labels.

\section{PREPROCESSING}

One frame of the SHL- challenge dataset is a 5 second segment. From SHL training-set and SHL validation-set, the frames consisting of multiple activities were excluded from learning. We excluded from SHL training-set and SHL validation-set the segments that are not the same activity label from learning. The number of excluded 
frames was 581 in SHL training-set and 104 in SHL validationset. We excluded these frames, but there were few change of the activity ratio. In addition, SHL training-set Hips had one frame that contained the Not a Number value in the sensor value, so it was excluded.

SHL Challenge set is sensor data in the terminal coordinate system. The values of Acc, Gyr and Mag were converted into the world coordinate system (North-East-Down Coordinate) by obtaining the rotation matrix from the orientation. Since the same axis always points up, it is possible to determine the field is coming from above or below. It is possible to reduce the influence of the difference in phone location and terminal direction. The conversion formula to the world coordinate system is shown below [3]. The rotation matrix $R_{N B}$ was created from the quaternion $\left[q_{w}, q_{x}, q_{y}, q_{z}\right]$ and transformed.

$$
\begin{aligned}
& R_{N B}=\left[\begin{array}{ccc}
1-2\left(q_{y}^{2}+q_{z}^{2}\right) & 2\left(q_{x} q_{y}-q_{w} q_{z}\right) & 2\left(q_{z} q_{z}-q_{w} q_{y}\right) \\
2\left(q_{x} q_{y}+q_{w} q_{z}\right) & 1-2\left(q_{x}^{2}+q_{z}^{2}\right) & 2\left(q_{y} q_{z}-q_{w} q_{x}\right) \\
2\left(q_{x} q_{z}-q_{w} q_{y}\right) & 2\left(q_{y} q_{z}+q_{w} q_{x}\right) & 1-2\left(q_{x}^{2}+q_{y}^{2}\right)
\end{array}\right] \\
& {\left[\begin{array}{c}
x \\
y \\
z
\end{array}\right]_{N}=R_{N B}\left[\begin{array}{c}
x \\
y \\
z
\end{array}\right]_{B}}
\end{aligned}
$$

\section{ESTIMATING USER OF SHL TEST-SET}

SHL test-set is not continuous every frame. Furthermore, participants are not informed what frames are User-2 or User-3. We thought the purpose of shuffling is what participants should classify activities from a 5 second time-window. Therefore, our team did not sort SHL test-set along with time series, classified from every frame. We estimated that what frame in SHL test-set is which user, User-2 or User-3, from SHL validation-set. SHL validation-set is a part of the SHL dataset that has already distributed. So, what frame is User-2 or User-3 can be specified from sensor data in the SHL dataset.

From the SHL dataset, we specified that the first half of SHL validation-set is user-2, and the latter half is User-3. We made a $\mathrm{CNN}$ model for estimating which user in SHL test-set. Figure 1 is the $\mathrm{CNN}$ configuration. $\mathrm{N}$ was set to 2 because it is a user classification.

The structure of $\mathrm{CNN}$ is as follows. First, the combination of four sets of two convolutional layers and a max pooling layer was applied to six time-frequency spectrums. Batch-normalization and Dropout $(0.2)$ were applied alternately with four sets of two convolutional layers and a max pooling layer. After that, they were connected by the fully connected layer and output by the soft-max function to obtain the $\mathrm{N}$ output probabilities. The relu function was used as the activation function on the way. We used TensorFlow's Keras API as a backend.

The used sensors were Acc, Mag, and Gyr converted to the world coordinate system. Time-frequency spectrums were created for each sensor on axis-z and the norm of a composite vector with axis- $x$ and axis-y. The FFT time-window was 640 milliseconds (64 points), and the overlap was 80 milliseconds, and the frames were standardized along the time axis. The time-frequency spectrum was used for the features.

Considering the ratio of eight activities, we split frames of User-2, and User-3 into 7:3 along with time series. Figure 2 is a confusion matrix, that a model learned $70 \%$ of SHL validation-set, and evaluated $30 \%$ of it. Then, input SHL test-set into this CNN model, and estimated user of each frame.

\section{ESTIMATING PHONE LOCATION IN SHL TEST-SET}

Participants were not informed of SHL test-set phone location. Therefore, as to increase F-measure for SHL test-set, there was a need to identify SHL test-set phone location. Moreover, we attempted to identify the phone location using a time-frequency spectrum.

First, we used a gradient-boosting algorithm, the basis on a decision tree, eXtream Gradient Boosting (XGBoost), and make a model to classify eight activities. The sensors that we used are LAcc, Gyr, and Mag sensors (world coordinate system). Feature values are below. Some feature values were standardized after the calculated mean and variance of every user. The Standardization frame is noted as (Standardization).

- Mean and variance of axis-x and y norm of LAcc (Standardization)

- Mean and variance of axis-z of LAcc (Standardization)

- Skewness and kurtosis of axis-z of LAcc (Standardization)

- Sums of FFT results of LAcc axis-z splitted by every $5 \mathrm{~Hz}$ (Standardization)

- The maximum values of FFT results of LAcc axis-z splitted by every $5 \mathrm{~Hz}$ (Standardization)

- Frequencies when FFT results of LAcc axis-z splitted by every $5 \mathrm{~Hz}$ get a maximum value

- Sums of FFT results of Gyr axis-z splitted by every $5 \mathrm{~Hz}$ (Standardization)

- The maximum values of FFT results of Gyr axis-z splitted by every $5 \mathrm{~Hz}$ (Standardization)

- Frequencies when FFT results of Gyr axis-z splitted by every $5 \mathrm{~Hz}$ get a maximum value

- Sums of FFT results of Mag axis-z splitted by every $5 \mathrm{~Hz}$ (Standardization)

- The maximum values of FFT results of Mag axis-z splitted by every $5 \mathrm{~Hz}$ (Standardization)

- Frequencies when FFT results of Mag axis-z splitted by every $5 \mathrm{~Hz}$ get a maximum value

In XGBoost, hyperparameters were adjusted, and finally the next hyperparameter was selected.

- max_depth $=16$

- min_child_weight $=7$

- learning_rate $=0.01$

- gamma $=0.005$

- sub_sample $=0.9$

- colsample_bytree $=0.8$

- n_estimator $=10000$

- early_stopping_rounds $=30$

We estimated SHL test-set's activity by the XGBoost model that learned SHL training-set and SHL validation-set. Secondly, we assumed that activities, which were "Walking," "Run," and "Car" is easy to identify the phone location. Then, the frames with an output probability of $75 \%$ or more for "Walking," "Run," and "Car" were 


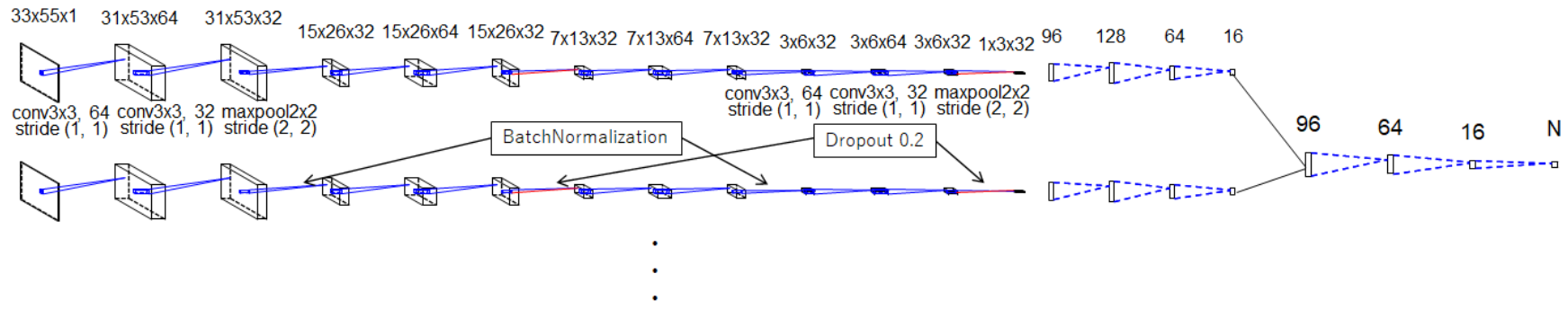

Figure 1: time-frequency $\mathrm{CNN}$ configuration estimating user, phone location and activity

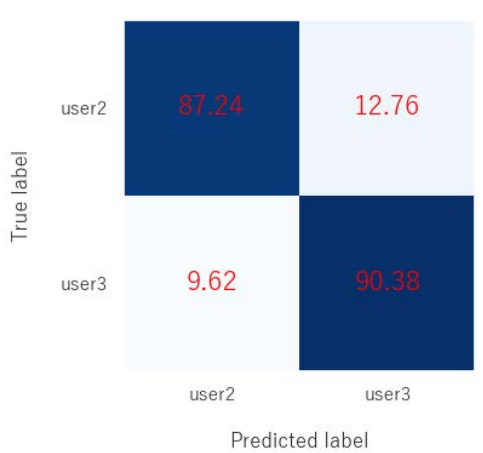

Figure 2: Confusion matrix of User estimation (SHL validation-set $\mathbf{3 0 \%}$ )

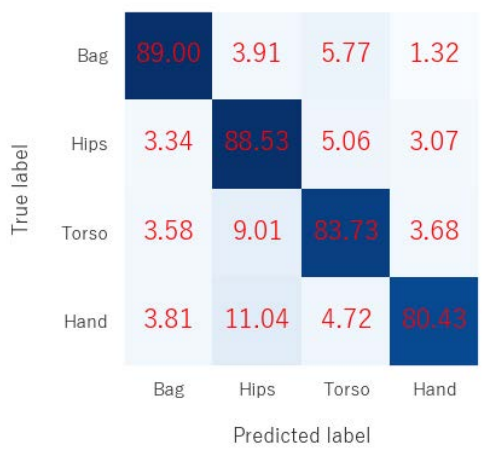

Figure 3: Confusion matrix of phone location estimation (SHL validation-set 30\%)

picked up. Secondly, we built the multi-input CNN that estimates the phone location same as user estimation (Fig.1). N was set to 4 because estimated phone location (Bag, Hips, Torso, Hand).

As the feature values, the time-frequency spectrums of axis- $\mathrm{z}$ and the norm of a composite vector with axis-x and axis-y. The used sensors were Acc, Mag, and Gyr (terminal coordinate system). FFT time-window is 640 milliseconds, and overlap is 80 milliseconds in the time-frequency spectrum. Figure 3 is a confusion matrix for a model that learned SHL training-set and evaluated by SHL validation-set. Moreover, we inputted frames that show over $75 \%$ output probability of "Walking," "Run," or "Car" into that CNN model and estimated phone location. Then, we extracted SHL testset frames with the maximum value of soft-max function output of 0.75 or more from the results, and checked the estimated labels. Since $79 \%$ of the extracted frames were estimated to be "Hips," our team decided to treat SHL test-set as "Hips."

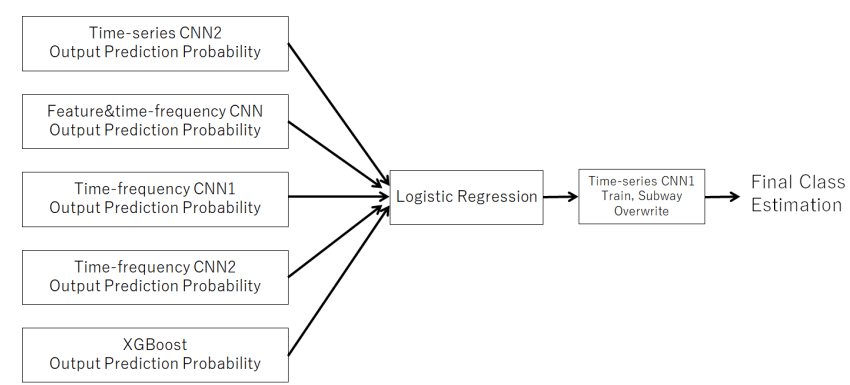

Figure 4: The flow of ensemble learning

\section{METHOD}

We classify activities using ensemble learning. Studies on activity recognition using ensemble learning have been conducted. Xu et al. constructed an ensemble learning model consisting of XGBoost, Random Forest, ExtraTrees, and soft-max Regression [4].

First, we created six different estimation models. The output probabilities of eight activities from six estimation models were given to logistic regression, and the final estimation result was given. SHL training-set (Only Hips) was used for training to create six different estimation models. SHL validation-set (Only Hips) was divided into 5:2:3 considering User-2 and User-3's ratio of labels in activity, respectively, and the first $50 \%$ was used for learning (our training data), and the middle $20 \%$ was used for early stopping (our validation data). The logistic regression model was made with the output probabilities of the eight activities output from the six estimation models as feature values. Figure 4 shows the flow of ensemble learning. The model used for the ensemble is as follows.

\subsection{Time-Series CNN}

This model was constructed using Pre and LAcc in the world coordinate system.

Figure 5 shows the flow of preprocessing. We applied with a 2 to $25 \mathrm{~Hz}$ band-pass filter to LAcc. As Lx and Ly, we calculated absolute values added the maximum value to the minimum value of each axis $x$ and $y$. Ln was the norm of a composite vector with axis- $x$ and axis-y. Ln2 was a feature value that we added a bigger one of Lx or Ly to Ln with each frame. About Pre, we applied with a high-pass filter through over $0.5 \mathrm{~Hz}$. The features were standardized in each of SHL training-set, SHL validation-set, and SHL test-set.

The time-series CNN1 trained all of SHL training-set and our training data. For time-series $\mathrm{CNN} 2$, the our training data User2 or -3 were trained from the time-series $\mathrm{CNN} 1$, therefore two models were created. The time-series CNN2 decided which of the 


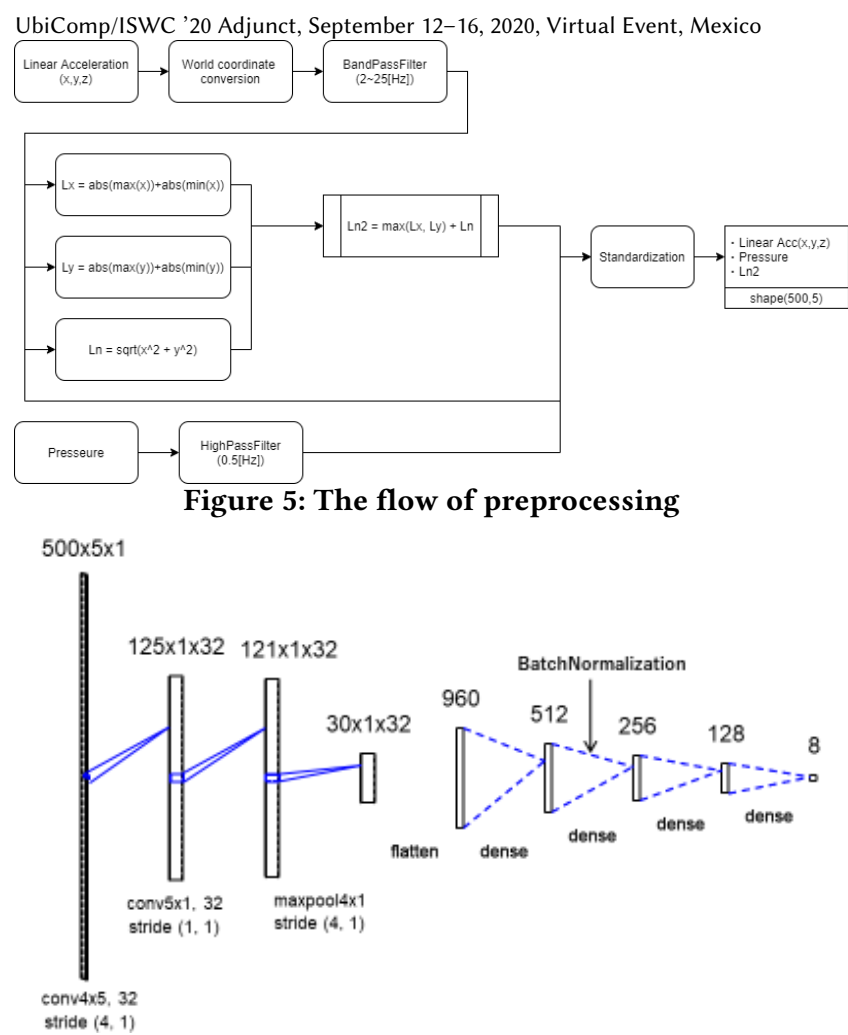

Figure 6: Time-series CNN configuration two models to estimate the activity by using the estimation result of the user of SHL test-set.

Figure 6 is the model of a 2D convolutional neural network. The model consisted of seven layers with two convolution layers, a max pooling layer, and four fully connected layers.

\subsection{Feature\&time-frequency $\mathrm{CNN}$}

This model was constructed using LAcc and Mag in the world coordinate system. In LAcc, we made values that was sum of continuous two points of all 500 points in each frame for a axis- $z$ and a norm of a composite vector with axis- $x$ and axis-y. In Mag, we made timefrequency spectrums for an axis- $z$ and a norm of a composite vector with axis- $x$ and axis-y. The FFT time-window was two seconds, and the overlap was 100 milliseconds.

Moreover, LAcc time-series and Mag time-frequency spectrums were standardized. SHL training-set has only User-1, this was standardized in each feature value. SHL validation-set and SHL test-set has two users, User-2 and -3. In each feature value of SHL validationset, this was standardized in each user. The SHL test-set was also standardized in data estimated as User-2 or -3 .

The model had two inputs, two 1D convolution layers for the input LAcc, a 2D convolution layer for the input Mag, four fully connected layers. Figure 7 shows the $\mathrm{CNN}$.

\subsection{Time-Frequency $\mathrm{CNN}$}

This model in Figure 1 was also used for this method. Since it is a classification of activity, $\mathrm{N}$ was set to 8 . We constructed a multi-input $\mathrm{CNN}$ with six time-frequency spectrums as inputs. The time-frequency spectrum used for input is the same as Chapter 4 .

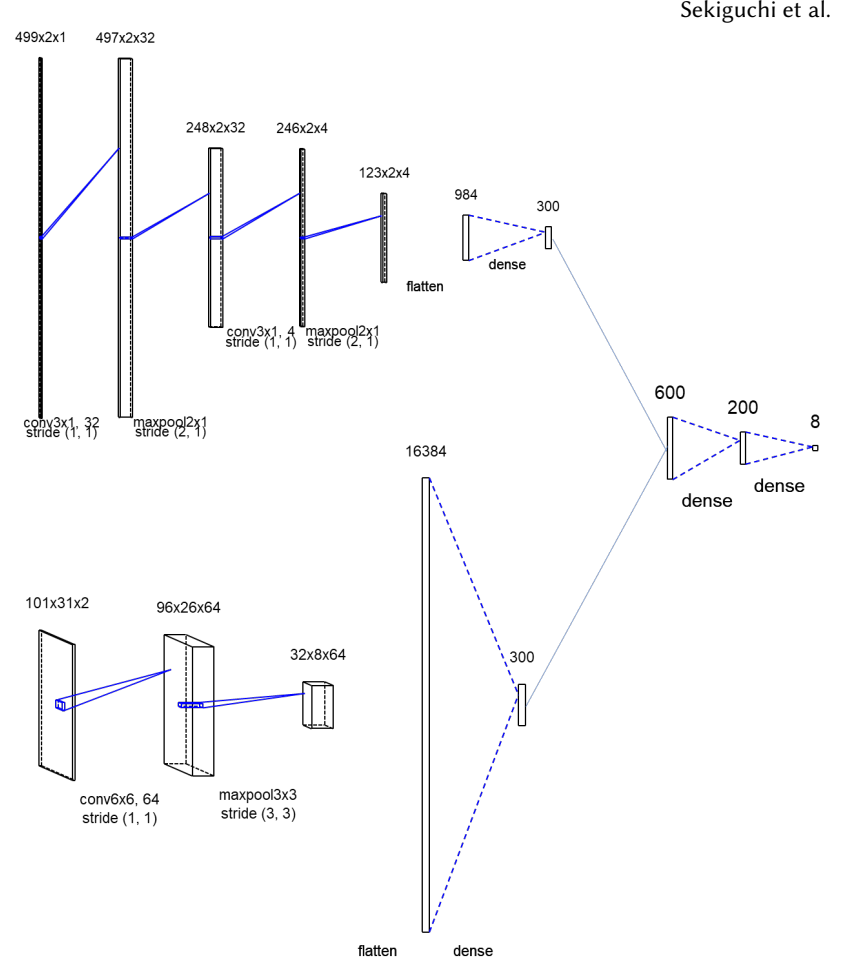

Figure 7: Feature\&time-frequency $\mathrm{CNN}$ configuration

It has been shown in past SHL Challenges that the time-frequency spectrum contributes to the activity recognition [5].

The time-frequency CNN1 trained all of SHL training-set and our training data. For time-frequency $\mathrm{CNN} 2$, our training data Users-2 or -3 were trained from time-frequency $\mathrm{CNN} 1$, then two models were created for user- 2 and -3 . Based on Chapter 4 estimation, we decided a estimation model from that two models.

\subsection{XGBoost}

This model was using XGBoost for classifying eight activities. The features and hyperparameters are the same as those described in Section 5. Used sensors, as feature values are LAcc, Gyr, and Mag in the world coordinate system. Previous studies have shown that the acceleration mean, variance, skewness, and kurtosis contribute to the estimation of activity [6].

\subsection{Ensemble Learning}

For ensemble learning, we used time-series CNN2, Feature\&timefrequency CNN, Time-frequency CNN1, 2 and XGBoost output probabilities as feature values. Our training data and our validation data were used to create the logistic regression model. SHL test-set was estimated by the logistic regression model trained by all SHL validation-set.

Next, the output labels of the logistic regression model were partially overwritten. The frame that time-series CNN1 estimated to be a train and subway was overwritten with it. Because the timeseries CNN1's estimation accuracy of train and subway was higher than the ensemble models. 
Ensemble Learning for Human Activity Recognition
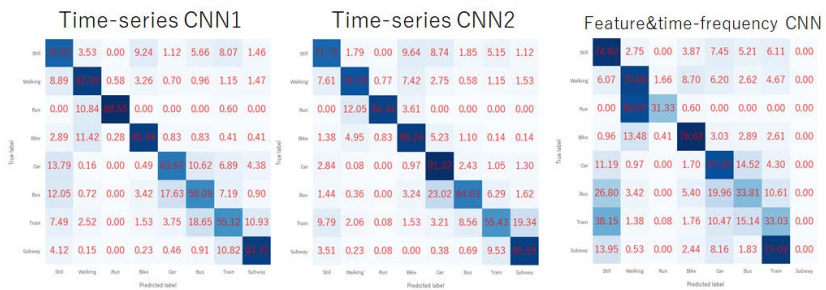

$X$ BBoost

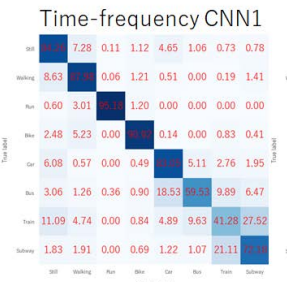

Time-frequency CNN2
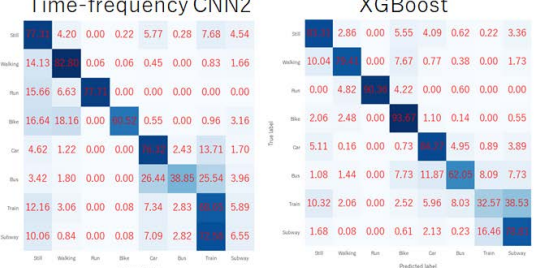

Figure 8: Six different confusion matrices for the last $30 \%$ of SHL validation-set

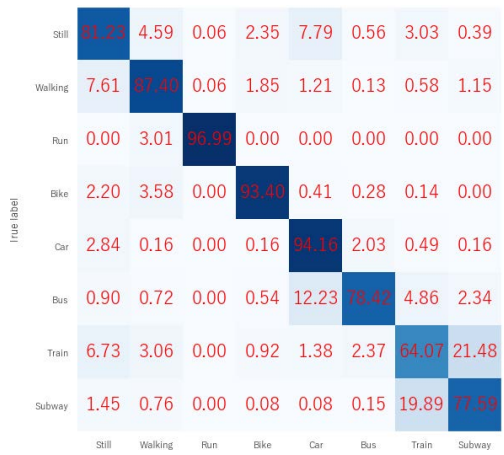

Figure 9: Confusion matrix for the last $30 \%$ of SHL validation-set

\section{RESULT}

Figure 8 shows the confusion matrix for the last $30 \%$ of SHL validationset in each model. Also in this result, the label overwriting of the frame estimated by Time-series CNN1 as train and subway is applied. Figure 9 shows the confusion matrix of the ensemble learning results of the last $30 \%$ SHL validation-set. SHL test-set was estimated by the logistic regression model that also learned the last $30 \%$ SHL validation-set. Then, the frame that time-series CNN1 estimated to be a train and subway was overwritten with it. Table 1 shows the F-measure of the models.

\section{CONCLUSION}

In this paper, we performed ensemble learning for activity recognition. We were able to challenge the estimation of the phone location of SHL test-set and the user. We estimated that SHL test-set's phone location was Hips, then we made six different models. The features for logistic regression model were output probabilities of Timeseries CNN2, Feature\&time-frequency CNN, Time-frequency CNN 1 and 2, and XGBoost. But in "Train" and "Subway", we used the estimation results of Time-series CNN1. In the final verification, a F-measure of $84.8 \%$ was obtained for last $30 \%$ SHL validation-set. From this result, it is expected that high accuracy will be obtained
UbiComp/ISWC '20 Adjunct, September 12-16, 2020, Virtual Event, Mexico

Table 1: The F-measure of the models (SHL validation-set $30 \%)$

\begin{tabular}{cc}
\hline Model name & F-measure \\
\hline \hline Time-series CNN1 & $72.5 \%$ \\
Time-series CNN2 & $76.0 \%$ \\
Feature\&time-frequency CNN & $46.1 \%$ \\
Time-frequency CNN1 & $76.5 \%$ \\
Time-frequency CNN2 & $62.0 \%$ \\
XGBoost & $74.5 \%$ \\
\hline Logistic regression & $83.9 \%$ \\
Logistic regression (Overwrite) & $84.8 \%$ \\
\hline
\end{tabular}

Table 2: The detail of the models created in this challenge

\begin{tabular}{ccl}
\hline Model name & Model size & Learning time \\
\hline \hline Time-series CNN & $5.4 \mathrm{MB}$ & 2 hours \\
Feature\&time-frequency CNN & $21.1 \mathrm{MB}$ & 2 hours \\
Time-frequency CNN & $6.9 \mathrm{MB}$ & 8 hours \\
XGBoost & $80.5 \mathrm{MB}$ & 20 minutes \\
\hline
\end{tabular}

for the estimation of the activities of SHL test-set. The recognition result for the testing dataset will be presented in the summary paper of the challenge [7].

\section{COMPUTER RESOURCES}

CPU core i7, 3.19GHz, 6 core, 12 threads, RAM 32GB, Geforce RTX 2060 super. Table 2 shows the learning time of the models created in this challenge.

\section{REFERENCES}

[1] H. Gjoreski, M. Ciliberto, L. Wang, F. J. Ordonez Morales, S. Mekki, S. Valentin, and D. Roggen. The university of sussex-huawei locomotion and transportation dataset for multimodal analytics with mobile devices. IEEE Access, 6:42592-42604, 2018.

[2] L. Wang, H. Gjoreski, M. Ciliberto, S. Mekki, S. Valentin, and D. Roggen. Enabling reproducible research in sensor-based transportation mode recognition with the sussex-huawei dataset. IEEE Access, 7:10870-10891, 2019.

[3] Vito Janko, Mitja Lustrek, Nina Reščič, Miha Mlakar, Vid Drobnič, Matjaz Gams, Gašper Slapničar, Martin Gjoreski, Jani Bizjak, and Matej Marinko. A new frontier for activity recognition: The sussex-huawei locomotion challenge. pages 15111520, 102018.

[4] Shoujiang Xu, Qingfeng Tang, Linpeng Jin, and Zhigeng Pan. A cascade ensemble learning model for human activity recognition with smartphones. Sensors, 19(10):2307, May 2019.

[5] Chihiro Ito, Masaki Shuzo, and Eisaku Maeda. Cnn for human activity recognition on small datasets of acceleration and gyro sensors using transfer learning. In Adjunct Proceedings of the 2019 ACM International foint Conference on Pervasive and Ubiquitous Computing and Proceedings of the 2019 ACM International Symposium on Wearable Computers, UbiComp/ISWC '19 Adjunct, page 724-729, New York, NY, USA, 2019. Association for Computing Machinery.

[6] Keisuke Yoshida, Shogo Matsuno, and Minoru Ohyama. Highly accurate method for estimating movement state of smartphone users. In Communications and Signal Processing 2016. RISP International Workshop on Nonlinear Circuits, 032016.

[7] L. Wang, H. Gjoreski, M. Ciliberto, P. Lago, K. Murao, T. Okita, and D. Roggen. Summary of the sussex-huawei locomotion-transportation recognition challenge 2020. Proceedings of the 2020 ACM International Joint Conference and 2020 International Symposium on Pervasive and Ubiquitous Computing and Wearable Computers, 2020 